\title{
A Disintegrin and Metalloproteinase 9 Is Involved in Ectodomain Shedding of Receptor-Binding Cancer Antigen Expressed on SiSo Cells
}

\author{
Kenzo Sonoda and Kiyoko Kato \\ Department of Obstetrics and Gynecology, Graduate School of Medical Sciences, Kyushu University, Maidashi 3-1-1, \\ Higashi-ku, Fukuoka 812-8582, Japan \\ Correspondence should be addressed to Kenzo Sonoda; kenzo@med.kyushu-u.ac.jp
}

Received 16 April 2014; Revised 16 June 2014; Accepted 9 July 2014; Published 7 August 2014

Academic Editor: Dominique Alfandari

Copyright (C) 2014 K. Sonoda and K. Kato. This is an open access article distributed under the Creative Commons Attribution License, which permits unrestricted use, distribution, and reproduction in any medium, provided the original work is properly cited.

In several human malignancies, the expression of receptor-binding cancer antigen expressed on SiSo cells (RCAS1) is associated with aggressive characteristics and poor overall survival. RCAS1 alters the tumor microenvironment by inducing peripheral lymphocyte apoptosis and angiogenesis, while reducing the vimentin-positive cell population. Although proteolytic processing, referred to as "ectodomain shedding," is pivotal for induction of apoptosis by RCAS1, the proteases involved in RCAS1-dependent shedding remain unclear. Here we investigated proteases involved in RCAS1 shedding and the association between tumor protease expression and serum RCAS1 concentration in uterine cancer patients. A disintegrin and metalloproteinase (ADAM) 9 was shown to be involved in the ectodomain shedding of RCAS1. Given the significant correlation between tumor ADAM9 expression and serum RCAS1 concentration in both cervical and endometrial cancer as well as the role for ADAM9 in RCAS1 shedding, further exploration of the regulatory mechanisms by which ADAM9 converts membrane-anchored RCAS1 into its soluble form should aid the development of novel RCAS1-targeting therapeutic strategies to treat human malignancies.

\section{Introduction}

To date, over 150 scientific reports have been published that concern the biological functions and clinical significance of RCAS1. RCAS1 is a 639 amino acid, type II membrane protein with an $\mathrm{N}$-terminal transmembrane segment and a C-terminal coiled-coil structure that is involved in oligomer formation [1]. Since RCAS1 promotes tumor cell evasion of immune surveillance by inducing apoptosis in immune cells, including peripheral lymphocytes, and also remodels the cancer stromal microenvironment, RCAS1 is believed to contribute to tumor progression [2]. Clinically, RCAS1 expression is significantly higher in cancerous tissues relative to normal tissues [3], and its expression increases during the progression from precancerous lesions to cancer $[4,5]$. RCAS1 expression is associated with several clinicopathological parameters of human malignancies, including histological type, differentiation, tumor size, stage, depth of invasion, lymphovascular space involvement, lymph node metastasis, and positive peritoneal cytological results [6]. In addition, RCAS1 is a negative predictor of overall survival in 15 different kinds of cancers occurring in the brain, oral cavity, lung, pleural mesothelium, esophagus, stomach, bile duct, gallbladder, pancreas, colon, gastrointestinal mesenchyme, kidney, prostate, uterine cervix, and endometrium [2].

RCAS1 is shed in the serum and pleural effusion and as such may be a useful biomarker for human cancer due to its ability to predict the results of medical treatments $[7,8]$. During the conversion from a membrane-anchored to a shedded protein, RCAS1 undergoes proteolytic processing known as "ectodomain shedding" [9]. Ectodomain shedding affects the biological activity of membrane proteins such as growth factors, growth factor receptors, cell-adhesion molecules, and extracellular matrix proteins by altering their localization and mode of action [10]. For membrane-anchored growth factors, ectodomain shedding can convert them into diffusible factors and greatly influence their functions. The membrane-anchored form of Spitz, a transforming growth 
factor (TGF)- $\alpha$-like molecule that is an epidermal growth factor receptor (EGFR) ligand in Drosophila, is inactive but is activated following proteolytic cleavage to yield a soluble protein [11]. In contrast, membrane-anchored c-kit ligand [12] and ephrins [13] are fully functional, while their soluble forms exhibit little or no biological activity. RCAS1 induces apoptosis mainly via its shedded form and not the membrane-anchored form. Therefore, regulation of the conversion of membrane-anchored proteins into their soluble form would be an important way to modify the action of these molecules, including RCAS1. Accumulating evidence demonstrated a role for proteolytic enzymes such as matrix metalloproteinase (MMP), ADAM, and the closely related ADAM with thrombospondin motifs (ADAMTSs) in cancer development and progression [14, 15]. MMPs, ADAMs, and ADAMTSs play a crucial role during all stages of cancer progression, from initiation to metastatic spreading. Besides their role in shedding of plasma membrane-associated proteins and intracellular signaling, these proteases regulate growth factor activation, angiogenesis, inflammation, and apoptosis $[16,17]$.

Although RCAS1 induces apoptosis mainly after being converted to its shedded form, the proteases involved in this ectodomain shedding remain unclear. To understand more clearly the regulation of membrane-anchored RCAS1 conversion, we sought to (1) identify key proteases involved in RCAS1 shedding and (2) determine whether there is an association between tumor protease expression and serum RCAS1 concentration in cervical and endometrial cancer patients.

\section{Materials and Methods}

2.1. Cell Lines. The human uterine cervical adenocarcinoma cell line SiSo, human breast adenocarcinoma cell line MCF7, human chronic myelogenous leukemia cell line K562, and mouse embryo fibroblast $\mathrm{L}$ cells were maintained in RPMI 1640 medium supplemented with 100 units $/ \mathrm{mL}$ penicillin G, $100 \mu \mathrm{g} / \mathrm{mL}$ streptomycin, and $10 \%$ fetal bovine serum (FBS) (ICN Biomedical, Irvine, CA) in a humidified incubator $\left(37^{\circ} \mathrm{C}, 5 \% \mathrm{CO}_{2}\right)$. Both SiSo and MCF-7 cells express membrane-anchored RCAS1, but RCAS1 shedding is undetectable in MCF-7 culture supernatants [9]. RCAS1 expression and shedding are undetectable in K562 cells and L cells that express a putative RCAS1 receptor [1]. We established the SiSo cell line from uterine cervical adenocarcinoma [18] and the other cell lines were purchased from the American Type Culture Collection.

2.2. Patients and Surgical Specimens. Tissue samples from cervical and endometrial cancer patients were used for immunohistochemical analysis. All patients had received medical treatment between April 2010 and January 2013 at the Department of Obstetrics and Gynecology, Kyushu University Hospital (Table 1). The mean patient age was 43 years (range of 24-81 years) for cervical cancer and 58 years (range of 37-84 years) for endometrial cancer. The histologic subtypes were 33 cases of squamous cell carcinoma and 14
TABLE 1: Clinicopathologic variables for uterine cancer patients.

\begin{tabular}{lc}
\hline Clinicopathologic variables & Number of patients \\
\hline Cervical cancer & \\
Age (years; mean \pm SD) & $43 \pm 11$ \\
Stage & 36 \\
$\quad$ I & 11 \\
$\quad$ II & \\
Histologic subtype & 33 \\
$\quad$ Squamous cell carcinoma & 14 \\
$\quad$ Adenocarcinoma & \\
Endometrial cancer & $58 \pm 11$ \\
Age (years; mean \pm SD) & \\
Stage & 28 \\
I & 5 \\
II & 13 \\
III & 2 \\
IV & \\
Grade & \\
1 & 24 \\
2 & 15 \\
3 & 9 \\
\hline
\end{tabular}

cases of adenocarcinoma in cervical cancer and 48 cases of endometrioid adenocarcinoma (24 cases of grade 1; 15 cases of grade 2; 9 cases of grade 3) in endometrial cancer. Cases were classified into stages as follows: 36 cases: stage I; 11 cases: stage II in cervical cancer; 28 cases: stage I; 5 cases: stage II; 13 cases: stage III; 2 cases: stage IV in endometrial cancer. These specimens were graded according to the 2008 International Federation of Gynecology and Obstetrics criteria. All specimens were fixed, embedded in paraffin, and stained with hematoxylin and eosin for determination of histologic subtype. Informed consent was obtained from all patients in this study. This study protocol was approved by the Ethical Committee of Kyushu University.

2.3. Evaluation of RCAS1 Expression by Flow Cytometry. To evaluate RCAS1 expression, flow cytometric analysis was performed using the monoclonal antibody 22-1-1 (MBL, Nagoya, Japan) that recognizes human RCAS1. Briefly, cells were harvested followed by incubation with 22-1-1 antibody on ice for 45 minutes. After the cells were washed, they were incubated for 45 minutes with fluorescein isothiocyanateconjugated goat anti-mouse IgM antibody (Pierce, Rockford, IL) on ice. The cells were again washed, and flow cytometric analysis was performed using FACScan (Becton Dickinson, San Jose, CA).

2.4. Enzyme-Linked Immunosorbent Assay (ELISA). We measured RCAS1 concentrations of the cell culture supernatant in triplicate with an ELISA kit (MBL, Nagoya, Japan), according to the manufacturer's instructions. The RCAS1 ELISA kit was applied in earlier investigations of the clinical significance of RCAS1 in uterine cancer [19]. The sensitivity of the RCAS1 
assay was $0.008 \mathrm{U} / \mathrm{mL}$. Mean concentrations of triplicate measurements were calculated.

2.5. Generation of SiSo Cells Expressing ADAM9 Small Interfering Ribonucleic Acid (siRNA). To construct specific siRNA for ADAM9, oligonucleotides were synthesized and purified by Takara Bio (Shiga, Japan) as follows: sense $5^{\prime}$-GGAGAUUUGGACCAAUGGATT- $3^{\prime}$ and antisense $5^{\prime}$ UCCAUUGGUCCAAAUCUCCTT- $3^{\prime}$. The target specificity of these sequences was confirmed by a BLAST search (http://www.ncbi.nlm.nih.gov/gene/). Homologous siRNA oligonucleotides were dissolved in buffer $(100 \mathrm{mM}$ potassium acetate, $30 \mathrm{mM}$ 4-(2-hydroxyethyl)-1-piperazineethanesulfonic acid (HEPES) plus potassium hydroxide, $2 \mathrm{mM}$ magnesium acetate, $\mathrm{pH} 7.4$ ) to a final concentration of $20 \mu \mathrm{M}$, heated to $90^{\circ} \mathrm{C}$ for 60 seconds, and incubated at $37^{\circ} \mathrm{C}$ for 60 minutes before use to disrupt higher order aggregates formed during synthesis. The complexes of transfection reagent (Invitrogen, Carlsbad, CA) plus siRNA were added to SiSo culture dishes. Assays were performed 48 hours after treatment. A nontargeting control siRNA that did not have homology with known gene targets in mammalian cells was also used. The control siRNA GC content was $38.1 \%$, which is identical to that of the siRNAs constructed here.

2.6. Generation of MCF-7 Cells Stably Expressing ADAM9. MCF-7 cells were transfected with the expression vector pEF-BOS carrying human ADAM9 complementary deoxyribonucleic acid (cDNA) or an empty pEF-BOS vector using Lipofectamine 2000 reagent (Invitrogen). Transfected cells were selected with $250 \mu \mathrm{g} / \mathrm{mL}$ G418. A clone was established after transfection with ADAM9 cDNA that was named MCF7/ADAM9. One clone was isolated after transfection with the pEF-BOS vector alone.

2.7. Western Blot Analysis. Cells were lysed in radioimmunoprecipitation assay buffer ( $1 \%$ Triton-X, $1 \%$ sodium deoxycholate, $0.1 \%$ sodium dodecyl sulfate (SDS), $150 \mathrm{mM} \mathrm{NaCl}$, $50 \mathrm{mM}$ Tris ( $\mathrm{pH} 8.0$ ), $0.2 \mathrm{unit} / \mathrm{mL}$ aprotinin, $2 \mu \mathrm{g} / \mathrm{mL}$ leupeptin, $1 \mu \mathrm{g} / \mathrm{mL}$ pepstatin A, $2 \mathrm{mM}$ phenylmethylsulfonyl fluoride, and $1 \mathrm{mM}$ sodium orthovanadate). Extracts were then subjected to SDS-polyacrylamide gel electrophoresis (PAGE) and immunoblotting analysis after transfer to Immobilon-P transfer membranes (Millipore Corporation, Bedford, MA). Membranes were probed with several antibodies including rabbit anti-ADAM9 (Chemicon, Temecula, CA) and mouse anti- $\beta$-actin (Novus Biologicals, Littleton, CO) antibodies. Peroxidase-conjugated goat anti-rabbit (Southern Biotech, Birmingham, AL) or anti-mouse IgG (Chemicon) was used as a secondary antibody.

2.8. Microarray Analysis. Hybridization targets for the GeneChip Human Gene 1.0 ST array were prepared using the GeneChip WT cDNA Synthesis and Amplification Kit, GeneChip Sample Cleanup Module and GeneChip WT Terminal Labeling Kit according to the manufacturer's protocol (Affymetrix, Santa Clara, CA). Briefly, the cells were harvested in the logarithmic growth phase and total RNA was extracted. Total RNA (100 ng) was converted into doublestranded cDNA (1st-cycle), and the complementary RNA (cRNA) was synthesized by in vitro transcription. After purification and measurement of cRNA, $10 \mu \mathrm{g}$ was converted into single-stranded DNA (ssDNA, 2nd cycle), of which $5.5 \mu \mathrm{g}$ was fragmented and labeled. The ssDNA was hybridized to the array described above for 16 hours at $45^{\circ} \mathrm{C}$. Following hybridization, the array was automatically washed and stained with the GeneChip Hybridization, Wash and Stain Kit. The Probe Array was scanned using the GeneChip Scanner 3000 7G. Microarray analysis was performed three times using three independent cell cultures.

2.9. Evaluation of Apoptotic Cell Death. Induction of apoptosis in K562 cells was evaluated by coculturing with four effecter cell types, including SiSo, MCF-7, MCF-7/ADAM9, or L cell. Each effecter cell $\left(1 \times 10^{5}\right.$ cells/well $)$ and K562 target cells were coincubated in a 6-well plate at 1-20:1 effecter/target (E/T) ratio. To enhance tight cell-cell contacts, the plates were centrifuged once after coculture initiation. The suspended cells were harvested and stained with the Annexin V-PE apoptosis detection kit (MBL) on days 14 after beginning the experiment. Flow cytometric analysis was performed to measure the number of apoptotic cells. To discriminate K562 cells from effecter cells, K562 cells were stained using the green fluorescence cell linker PKH kit (Sigma, St. Louis, MI) before coculture initiation. Evaluation of K562 cell apoptosis was performed three times using three independent cell cultures.

2.10. Immunocytochemical Detection of RCAS1 and ADAM9 Association. A proximity ligation assay (PLA) to detect an association between RCAS1 and ADAM9 was carried out using a Duolink detection kit (Olink Bioscience, Uppsala, Sweden). Briefly, SiSo, MCF-7, ADAM9 siRNA-transfected SiSo, and RCAS1 siRNA-transfected SiSo cells [20] were seeded into 8 -well chamber slides. On the next day, cultures were fixed in $90 \%$ ethanol $/ 5 \%$ acetic acid and subjected to PLA. Slides were incubated with mouse anti-RCAS1 (MBL) and rabbit anti-ADAM9 (Chemicon) antibodies and then secondary antibodies conjugated to unique DNA probes (PLA probe MINUS and PLUS) were added. Ligation and circularization of the DNA were followed by a rolling circle amplification step, and reactions were detected by a complementary Tex613 fluorophore-labeled DNA linker [21]. Slides were evaluated using an LSM 510 META confocal microscope (Zeiss, Jena, Germany).

2.11. Immunohistochemistry. For immunohistochemical analyses, one or two representative samples selected for each case were analyzed by means of the streptavidin-biotin method. The 22-1-1 antibody (MBL) or rabbit anti-ADAM9 antibody (Abcam, Cambridge, MA) was applied as the primary antibody. Positive control samples were as follows: for RCAS1, cervical adenocarcinoma, which was used to manufacture the 22-1-1 antibody [3], and for ADAM9, breast cancer [22]. We also performed assays without immunized mouse and rabbit immunoglobulins as negative controls. No 
TABLE 2: Microarray data on proteases.

\begin{tabular}{|c|c|c|c|c|c|}
\hline Gene symbol & Probe ID & Probe set ID & SiSo signal & MCF-7 signal & SiSo/MCF-7 \\
\hline ADAM2 & HU133p2_17106 & 207664_at & 7.0 & 6.3 & 1.11 \\
\hline ADAM3A & HU133p2_26379 & 217090_at & 1.4 & 1.9 & 0.74 \\
\hline ADAM5 & HU133p2_26289 & 216998_s_at & 2.9 & 7.2 & 0.40 \\
\hline ADAM6 & HU133p2_47159 & 237909_at & 3.1 & 7.1 & 0.44 \\
\hline ADAM7 & HU133p2_20597 & 211239_s_at & 7.3 & 3.0 & 2.43 \\
\hline ADAM8 & HU133p2_14627 & 205179_s_at & 12.3 & 8.4 & 1.46 \\
\hline ADAM9 & HU133p2_11830 & 202381_at & 1856.6 & 275.1 & 6.75 \\
\hline ADAM10 & HU133p2_06424 & 1562137_at & 5.2 & 4.7 & 1.11 \\
\hline ADAM11 & HU133p2_49087 & 239837_at & 9.6 & 8.2 & 1.17 \\
\hline ADAM12 & HU133p2_51710 & 242460_at & 9.2 & 6.4 & 1.44 \\
\hline ADAM15 & HU133p2_26298 & 217007_s_at & 83.6 & 43.4 & 1.93 \\
\hline ADAM17 & HU133p2_22834 & 213532_at & 117.7 & 195.5 & 0.60 \\
\hline ADAM18 & HU133p2_17039 & 207597_at & 5.6 & 8.3 & 0.67 \\
\hline ADAM19 & HU133p2_30412 & 221128_at & 8.9 & 13.3 & 0.67 \\
\hline ADAM20 & HU133p2_16866 & 207423_s_at & 7.7 & 18.7 & 0.41 \\
\hline ADAM21 & HU133p2_17107 & 207665_at & 6.8 & 6.2 & 1.10 \\
\hline ADAM22 & HU133p2_53445 & 244194_at & 5.9 & 5.6 & 1.05 \\
\hline ADAM23 & HU133p2_15493 & 206046_at & 6.2 & 24.0 & 0.26 \\
\hline ADAM28 & HU133p2_17694 & 208269_s_at & 1.6 & 7.4 & 0.22 \\
\hline ADAM29 & HU133p2_30621 & 221337_s_at & 0.4 & 9.1 & 0.04 \\
\hline ADAM30 & HU133p2_30730 & 221446_at & 3.7 & 9.5 & 0.39 \\
\hline ADAM32 & HU133p2_00020 & 1552266_at & 11.3 & 10.4 & 1.09 \\
\hline ADAM33 & HU133p2_43119 & 233868_x_at & 33.1 & 31.9 & 1.04 \\
\hline ADAMTS1 & HU133p2_31443 & 222162_s_at & 0.6 & 4.4 & 0.14 \\
\hline ADAMTS2 & HU133p2_23835 & 214535_s_at & 3.6 & 2.3 & 1.57 \\
\hline ADAMTS3 & HU133p2_24209 & 214913_at & 4.5 & 5.3 & 0.85 \\
\hline ADAMTS4 & HU133p2_02285 & 1555380_at & 13.9 & 16.1 & 0.86 \\
\hline ADAMTS5 & HU133p2_29220 & 219935_at & 2.2 & 2.4 & 0.92 \\
\hline ADAMTS6 & HU133p2_09800 & 1570351_at & 3.5 & 8.2 & 0.43 \\
\hline ADAMTS7 & HU133p2_29991 & 220706_at & 2.2 & 5.9 & 0.37 \\
\hline ADAMTS8 & HU133p2_29962 & 220677_s_at & 8.4 & 12.4 & 0.68 \\
\hline ADAMTS9 & HU133p2_03368 & 1556989_at & 6.4 & 11.1 & 0.58 \\
\hline ADAMTS10 & HU133p2_41388 & 232133_at & 1.2 & 0.8 & 1.50 \\
\hline ADAMTS12 & HU133p2_30705 & 221421_s_at & 13.3 & 19.8 & 0.67 \\
\hline ADAMTS13 & HU133p2_33121 & 223844_at & 3.0 & 10.1 & 0.30 \\
\hline ADAMTS15 & HU133p2_00845 & 1553427_at & 2.0 & 1.5 & 1.33 \\
\hline ADAMTS16 & HU133p2_46339 & 237089_at & 2.2 & 2.4 & 0.92 \\
\hline ADAMTS17 & HU133p2_00332 & 1552725_S_at & 9.9 & 17.0 & 0.58 \\
\hline ADAMTS18 & HU133p2_00702 & 1553234_at & 9.1 & 10.7 & 0.85 \\
\hline ADAMTS19 & HU133p2_00663 & 1553180_at & 4.8 & 26.0 & 0.18 \\
\hline ADAMTS20 & HU133p2_30002 & 220717_at & 2.4 & 4.6 & 0.52 \\
\hline ADAMDEC1 & HU133p2_15581 & 206134_at & 0.5 & 4.5 & 0.11 \\
\hline ADAMTSL1 & HU133p2_33638 & 224371_at & 1.0 & 2.1 & 0.48 \\
\hline ADAMTSL2 & HU133p2_16076 & 206629_at & 1.2 & 1.2 & 1.00 \\
\hline ADAMTSL3 & HU133p2_04979 & 1559748_at & 2.2 & 12.0 & 0.18 \\
\hline MMP1 & HU133p2_13923 & 204475_at & 35.0 & 46.0 & 0.76 \\
\hline MMP2 & HU133p2_08387 & 1566677_at & 1.2 & 0.4 & 3.00 \\
\hline MMP3 & HU133p2_15276 & 205828_at & 19.3 & 25.0 & 0.77 \\
\hline
\end{tabular}


TABLE 2: Continued.

\begin{tabular}{|c|c|c|c|c|c|}
\hline Gene symbol & Probe ID & Probe set ID & SiSo signal & MCF-7 signal & SiSo/MCF-7 \\
\hline MMP7 & HU133p2_13707 & 204259_at & 17.9 & 5.4 & 3.31 \\
\hline MMP8 & HU133p2_16774 & 207329_at & 0.6 & 0.4 & 1.50 \\
\hline MMP9 & HU133p2_13384 & 203936_s_at & 21.2 & 43.1 & 0.49 \\
\hline MMP10 & HU133p2_15128 & 205680_at & 1.1 & 10.1 & 0.11 \\
\hline MMP11 & HU133p2_45158 & 235908_at & 2.3 & 14.4 & 0.16 \\
\hline MMP12 & HU133p2_14028 & 204580_at & 3.0 & 3.9 & 0.77 \\
\hline MMP13 & HU133p2_15407 & 205959_at & 7.9 & 10.5 & 0.75 \\
\hline MMP14 & HU133p2_09936 & 160020_at & 18.6 & 28.6 & 0.65 \\
\hline MMP15 & HU133p2_53134 & 243883_at & 1.2 & 1.6 & 0.75 \\
\hline MMP16 & HU133p2_17594 & 208166_at & 3.7 & 4.7 & 0.79 \\
\hline MMP17 & HU133p2_15681 & 206234_s_at & 6.9 & 28.0 & 0.25 \\
\hline MMP19 & HU133p2_14022 & 204574_s_at & 2.3 & 4.7 & 0.49 \\
\hline MMP20 & HU133p2_17041 & 207599_at & 1.9 & 0.9 & 2.11 \\
\hline MMP21 & HU133p2_00237 & 1552592_at & 3.8 & 0.8 & 4.75 \\
\hline MMP23A/B & HU133p2_16565 & 207118_s_at & 1.4 & 0.6 & 2.33 \\
\hline MMP24 & HU133p2_17809 & 208387_s_at & 5.0 & 5.7 & 0.88 \\
\hline MMP25 & HU133p2_49304 & 240054_at & 14.4 & 34.8 & 0.41 \\
\hline MMP26 & HU133p2_29826 & 220541_at & 4.1 & 7.7 & 0.53 \\
\hline MMP27 & HU133p2_30068 & 220783_at & 1.1 & 0.7 & 1.57 \\
\hline MMP28 & HU133p2_33479 & 224207_x_at & 14.8 & 23.3 & 0.64 \\
\hline MMPL1 & HU133p2_16735 & 207289_at & 8.7 & 9.6 & 0.91 \\
\hline
\end{tabular}

significant immunohistochemical reaction occurred in the control sections.

Immunohistochemical expression of RCAS1 and ADAM9 was reviewed without knowledge of the clinicopathologic data. Evaluation of expression consisted of an examination of five representative fields, with 1000 tumor cells (200 for each field) being counted via a microscope with a high-power $(400 x)$ objective. Tissue sections with more than $5 \%$ reactive cells were defined as positive and graded as follows: $1+, 5 \%$ to $25 \%$ positive cells; $2+, 26 \%$ to $50 \%$ positive cells; and $3+, 51 \%$ to $100 \%$ positive cells.

2.12. Statistical Analysis. The Fisher's exact (chi-square) test was done to evaluate the association between RCAS1 and ADAM9 expression in tumor tissues resected from uterine cancer patients. The Mann-Whitney test was performed to check differences in antigen expression and secretion between different groups of cells. $P$ values of $<0.05$ were considered statistically significant.

\section{Results}

3.1. Differences in Protease Expression between SiSo and MCF7 Cells. The expression of proteases was compared between SiSo and MCF-7 cells by microarray analysis (Table 2). The ADAM9 expression level was significantly higher in SiSo cells, as shown by relative signals of 1856.6 and 275.1 in SiSo and MCF-7 cells, respectively, which yields a relative ratio of 6.75. No other proteases showing strong expression signals were significantly different between SiSo and MCF-7 cells.
3.2. Changes in RCAS1 Expression and Shedding after Gene Transfection. ADAM9 expression was knocked down in SiSo cells with siRNA. ADAM9 siRNA-transfected cells showed suppressed ADAM9 expression and inversely increased RCAS1 expression on the cell surface (Figure 1(a) (A) (B)). The RCAS1 expression and concentration were also quantitatively analyzed and shown in Figure 1(a) (C). While transfection of ADAM9 siRNA significantly augmented RCAS1 expression, the amount of RCAS1 in the culture supernatant was markedly decreased $(P=0.0495)$. On the other hand, ADAM9 expression was upregulated in MCF-7 cells following transfection of ADAM9 cDNA (Figure 1(b) (A)). However, RCAS1 expression was significantly reduced, even though RCAS1 shedding was accelerated by induction of ADAM9 expression (Figure 1(b) (B) (C)) ( $P=0.0495)$. On the other hand, the ADAM17 expression level was also higher than other proteases. ADAM17 is expressed in various tissues and has been reported to be associated with cancer progression events such as invasion, migration, and metastasis [15]. We also evaluated RCAS1 expression and shedding after ADAM17 gene transfection and found no significant change in either expression or shedding (see Supplementary Figure 1 in Supplementary Material available online at http://dx.doi.org/10.1155/2014/482396). Taken together, these data indicate that ADAM9 rather than ADAM17 is involved in RCAS1 shedding.

3.3. Analysis of Apoptotic Cell Death Induced in K562 Cells. Apoptosis of K562 cells was induced using a coculture system 


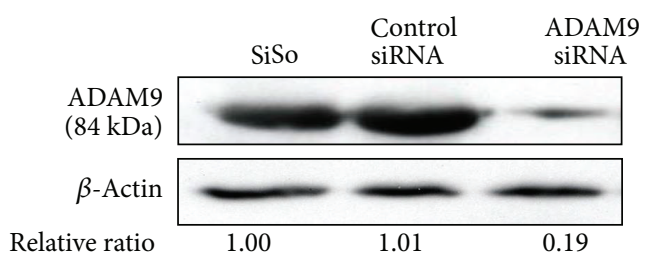

(A)
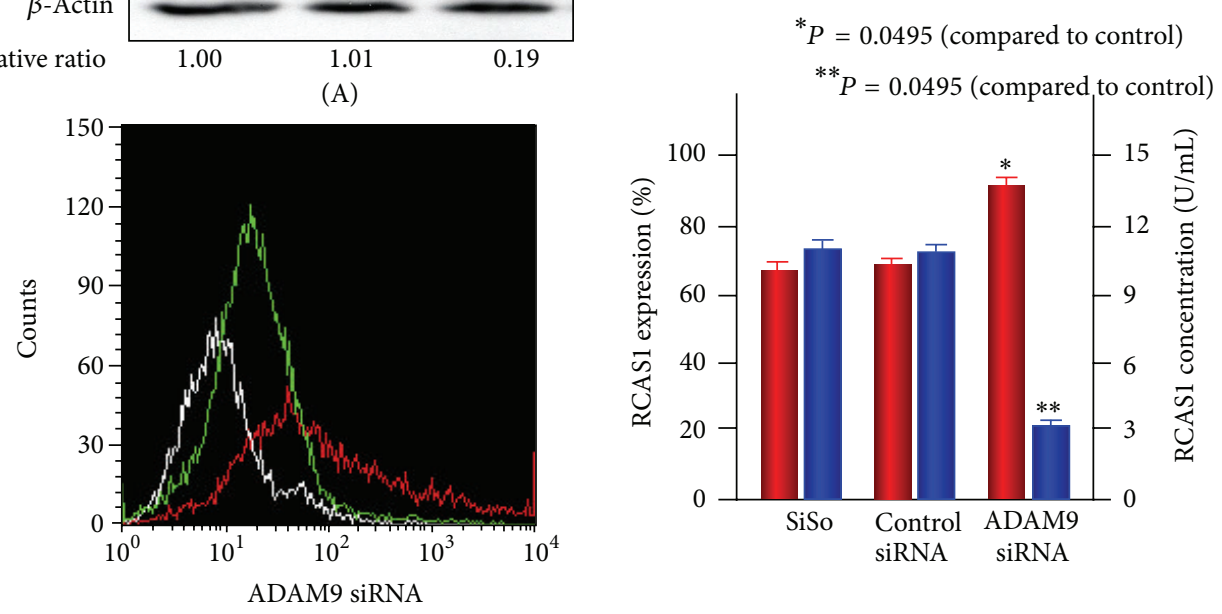

- Control IgM - ADAM9 siRNA
- Control siRNA

(B)

Expression

Concentration

(C)

(a)

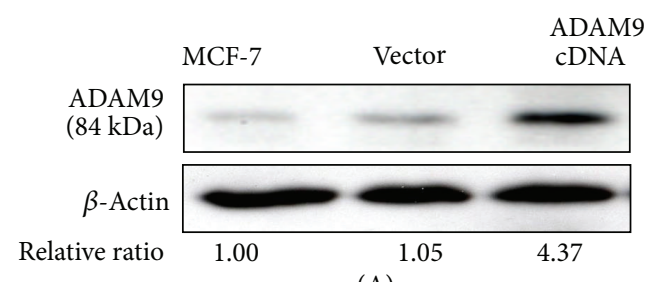

(A)
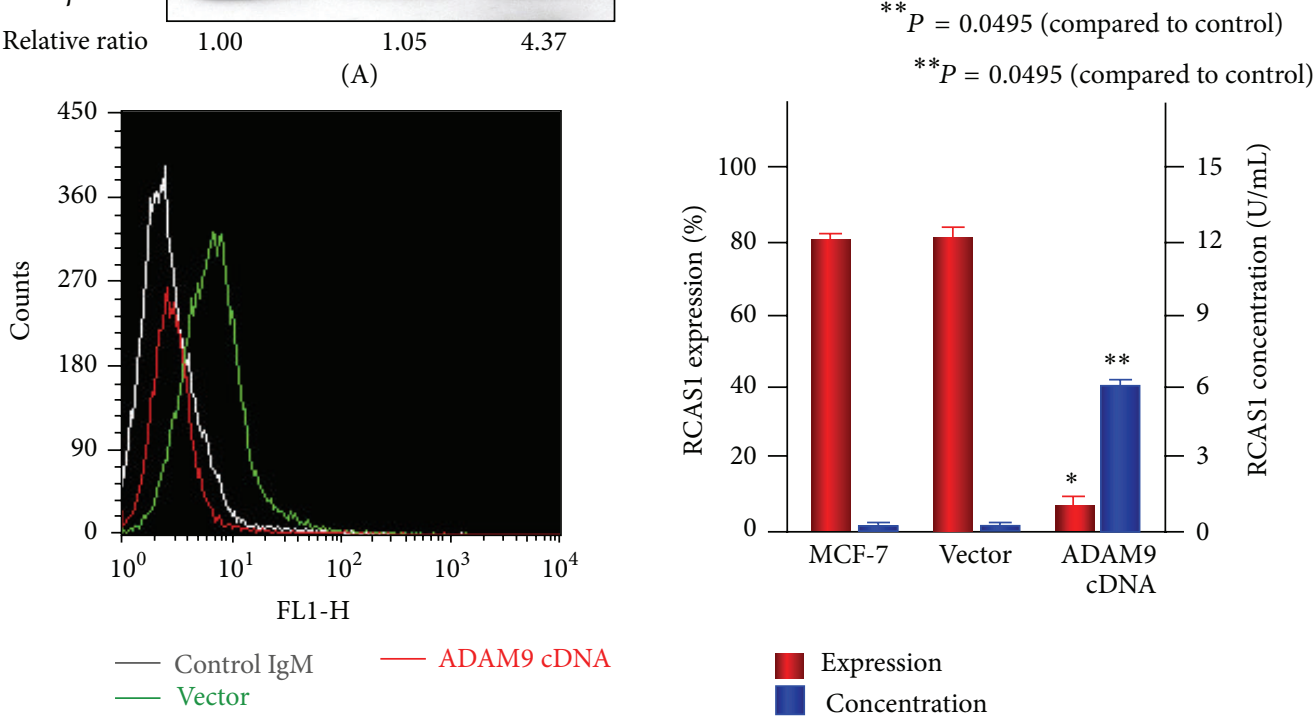

(B)

(C)

(b)

Figure 1: Change in RCAS1 expression and shedding after gene transfection. (a) ADAM9 siRNA transfection in SiSo cells. (A) A Western blot revealed that ADAM9 expression diminished after ADAM9 siRNA transfection. (B) Flow cytometric analysis showed that cell surface expression of RCAS1 increased after ADAM9 siRNA transfection. (C) Transfection of ADAM9 siRNA significantly augmented RCAS1 expression but decreased the RCAS1 concentration in culture supernatants $(P=0.0495)$. (b) ADAM9 cDNA transfection in MCF-7 cells. (A) A Western blot revealed that ADAM9 expression was enhanced after ADAM9 cDNA transfection. (B) Flow cytometric analysis showed that the cell surface expression of RCAS1 decreased after ADAM9 cDNA transfection. (C) Transfection of ADAM9 cDNA significantly diminished RCAS1 expression but increased the concentration of RCAS1 in culture supernatants $(P=0.0495)$. Mean values of triplicate measurements are shown. 
with SiSo, MCF-7, MCF-7/ADAM9, and L cells. Both L cells and MCF-7 cells were used as a negative control and SiSo cells were a positive control for inducing apoptosis in K562 cells [9]. Although K562 apoptosis was not induced by coculture with MCF-7 cells, the effecter cell MCF-7/ADAM9 could induce apoptosis in K562 cells. Figure 2(a) shows that the annexin- $\mathrm{V}$ positive ratio increased dependently on the $\mathrm{E} / \mathrm{T}$ ratio after 4 days of culture wherein $22.5 \%$ (this number represents the percentage of cells that were double positive for PKH2 and annexin-V) of K562 cells were apoptotic with a 20:1 E/T ratio. K562 apoptosis increased significantly depending on the culture period (Figure 2(b)) $(P=0.0495)$.

Next, we measured the RCAS1 concentration in cell supernatants. Although RCAS1 was not detected in L cells and MCF-7 cells, MCF-7/ADAM9 cells shed RCAS1 depending on the length of the culture period (Figure 2(c)). The level of RCAS1 shed from MCF-7/ADAM9 cells was lower than for SiSo cells, but the amount of RCAS1 significantly increased after four days of culture as compared to the first day $(P=$ 0.0495). These results suggest that apoptosis of K562 cells was induced by RCAS1 that was shed after ADAM9 proteolysis.

3.4. RCAS1 and ADAM9 Expression in Cell Lines and Cancerous Tissues. RCAS1 and ADAM9 colocalization was immunocytochemically analyzed in SiSo and MCF-7 cells using a Duolink detection kit. Negative controls without immunized immunoglobulins showed no red dots indicating colocalization of RCAS1 and ADAM9 (Supplementary Figures 2(a) and 2(b)). While red dots were observed in SiSo cells, they were absent in MCF-7 cells (Figure 3(a) (A) (B)). Although red dots in ADAM9 siRNA-transfected SiSo cells and RCAS1 siRNA-transfected SiSo cells rarely occurred (Figure 3(a) (C) (D)), they were occasionally observed in MCF-7/ADAM9 cells (Supplementary Figure 2(e)).

RCAS1 and ADAM9 expression was also evaluated in cancerous tissues by immunohistochemistry. ADAM9 expression was detected in normal cervical epithelium and endometrial glands with weak cytoplasmic staining and membrane immunoreactivity (data not shown). On the other hand, prominent staining for ADAM9 was detected in cervical and endometrial cancer (Figure 3(b)). The difference in ADAM9 protein expression levels between normal epithelium and cancerous tissues was highly significant. Diffuse staining for RCAS1 and ADAM9 was observed both in the cytoplasm and on the cell membrane of cancer cells. Of 47 patients with cervical cancer, $8,17,13$, and 9 cases showed no expression, $1+, 2+$, and $3+$ of RCAS1, respectively, while 7,8 , 11 , and 21 cases had no expression, $1+, 2+$, and $3+$, respectively, for ADAM9. Two cases were double-negative and 34 cases were double-positive for RCAS1 and ADAM9. Five cases were single-positive for RCAS1 and 6 cases were single-positive for ADAM9. In 48 patients with endometrial cancer, 16, 14,12 , and 6 cases showed no expression, $1+, 2+$, and 3+, respectively, for RCAS1, while for ADAM9, 6, 9, 14, and 19 cases had no expression, $1+, 2+$, and $3+$, respectively. Two cases were double-negative and 28 cases were double-positive for RCAS1 and ADAM9. Four cases were single-positive for RCAS1 and 14 cases were single-positive for ADAM9. There was no statistically significant association between RCAS1 and ADAM9 expression in both cervical and endometrial cancer.

3.5. The Association between Serum RCAS1 Concentration and RCAS1/ADAM9 Expression in Uterine Cancer Patients. We evaluated the association between serum RCAS1 concentration and RCAS1/ADAM9 expression in 47 cervical and 48 endometrial cancer patients. Serum RCAS1 levels significantly increased in a manner that was dependent on RCAS1 and ADAM9 expression in both cancer types (Figures 4(a) and 4(b)). These data further support a role for ADAM9 in regulating RCAS1 shedding in human uterine cancer.

\section{Discussion}

This is the first report showing that ADAM9 is involved in RCAS1 ectodomain shedding. ADAM9 is a member of the ADAM protein family, for which 40 gene members have currently been identified with 21 members being functional in humans [23]. ADAMs are membrane-anchored glycoproteins that consist of pro- and metalloprotease, disintegrin, cysteine-rich, EGF-like, and cytoplasmic domains, which enable these proteins to have a versatile range of physiological and pathological functions [24]. Some ADAMs participate in fertilization, myogenesis, neurogenesis, and activation of growth factors/immune regulators such as tumor necrosis factor (TNF)- $\alpha$ [25]. On the other hand, specific ADAMs have been implicated in a number of diseases, including rheumatoid arthritis, Alzheimer's disease, atherosclerosis, asthma, and cancer [17, 26]. ADAM9 was cloned and sequenced by Weskamp et al. in 1996 [27]. ADAM9 is widely expressed in the human body and is a catalytically active metalloprotease-disintegrin protein that has been implicated in the ectodomain cleavage of heparin-binding (HB)-EGF and as an $\alpha$-secretase for the amyloid precursor protein [17]. Olson et al. demonstrated the reproductive stage-specific expression of ADAM9 mRNA in rabbit uterine epithelium during the peri-implantation period [28]. ADAM9 expression is upregulated as progesterone levels rise and at blastocyst implantation sites. ADAM9 also plays a pivotal role in some signaling pathways, wherein transmission of information might induce some inconvertible exacerbations of disease [29]. ADAM9 is reportedly involved in several human diseases such as inflammatory disorders, oxygeninduced retinopathy (OIR), and cancer [30]. ADAM9 expression was found to be upregulated in various solid tumors and is often associated with adverse prognostic parameters or shorter patient survival times. ADAM9 overexpression was reported in several human carcinomas, including oral [31], lung [32], breast [21, 33], stomach [34], liver [35], pancreas [36], colon [37], kidney [38], prostate [39], cervix [40], and melanoma [41], and is correlated with cancer progression and metastasis, as well as having a predictive capacity for patient survival times. The background for the clinical significance of ADAM9 in tumor progression has been investigated by in vitro experiments. ADAM9 expression was found to be elevated in a cell line having high metastatic potential as compared to cell lines that had a low metastatic potential [35]. 

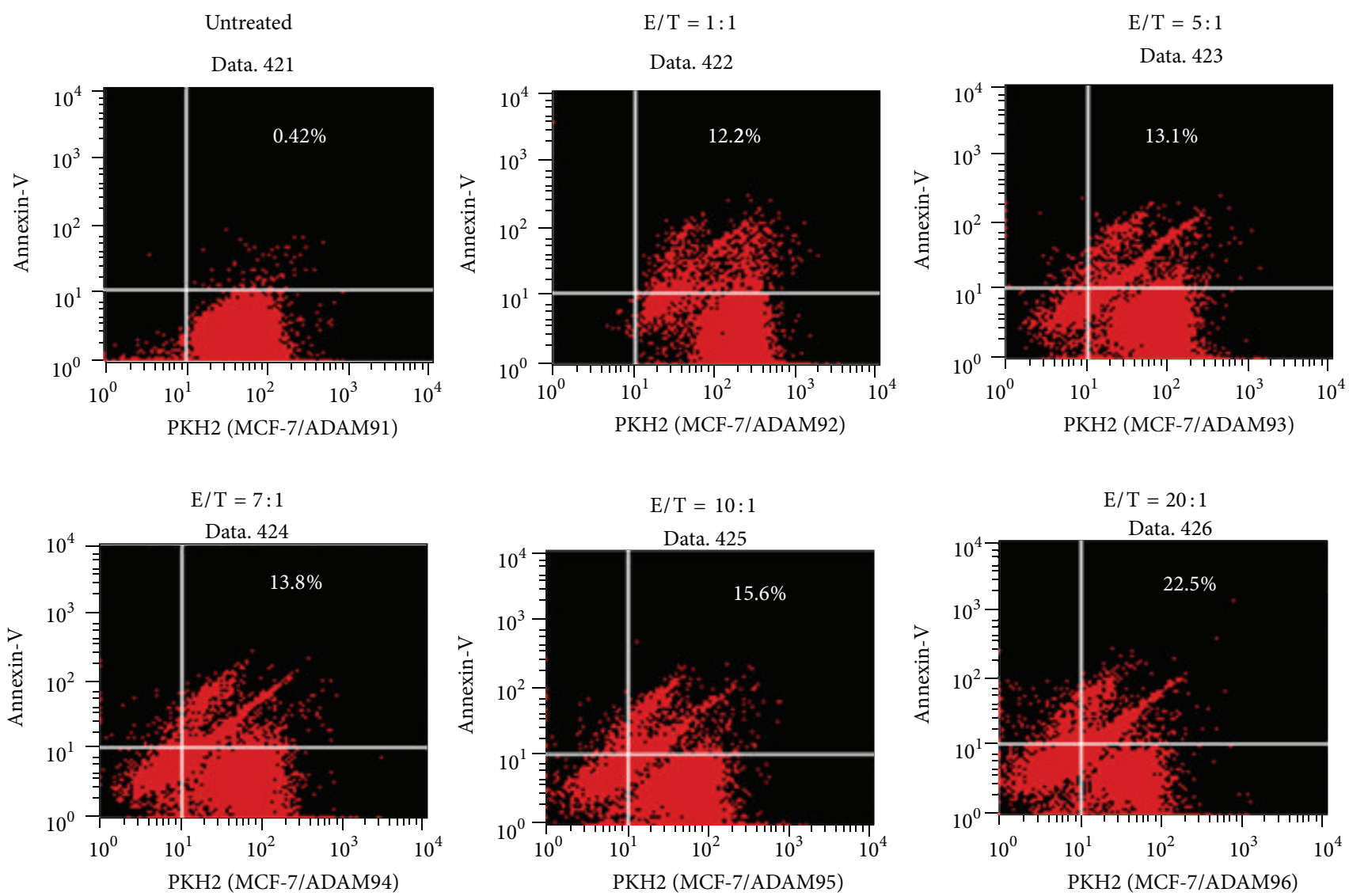

(a)

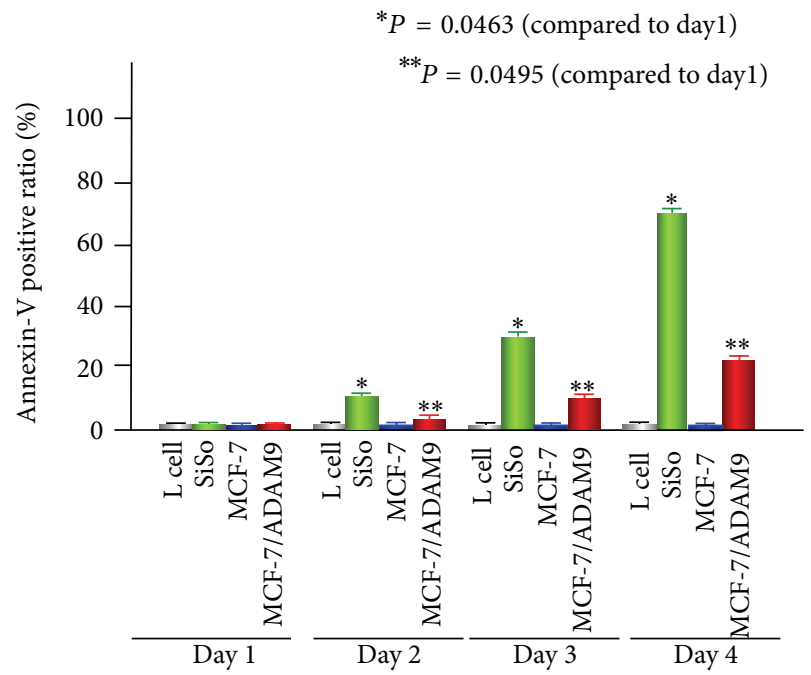

(b)

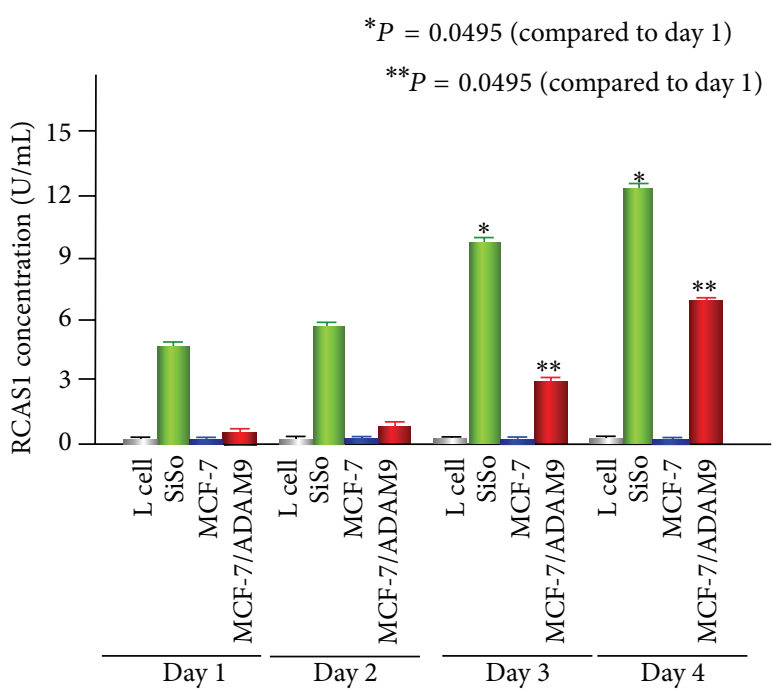

(c)

Figure 2: Analysis of apoptotic cell death induced in K562 cells. (a) K562 cell apoptosis was analyzed by flow cytometry. Increases in the annexin- $\mathrm{V}$ positive ratio were dependent on the E/T ratio after 4 days of culture. The percentage of cells double positive for PKH2 and annexin- $\mathrm{V}$ is indicated. (b) The increase in the number of apoptotic K562 cells was dependent on the culture period (E/T ratio = $20: 1)$. Both L cells and MCF-7 cells were used as a negative control and SiSo cells acted as a positive control for inducing apoptosis in K562 cells. (c) The RCAS1 concentration in cell supernatants was measured by ELISA. The RCAS1 level in MCF-7/ADAM9 supernatants increased with the culture time, even though MCF-7 cells alone do not shed RCAS1. Mean values of triplicate measurements are shown. 


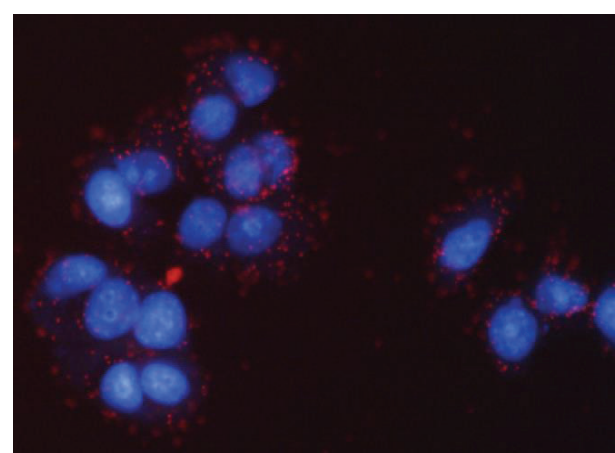

(A) SiSo

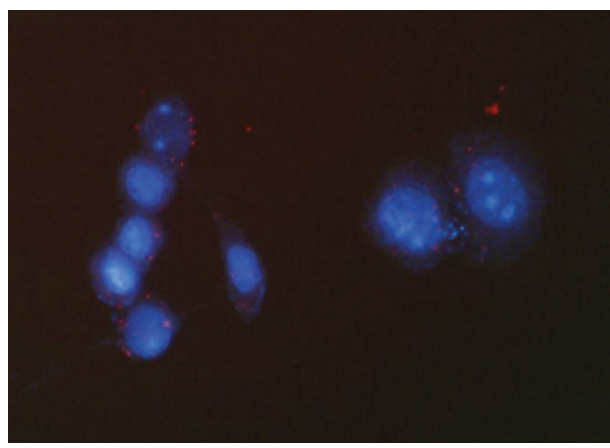

(C) ADAM 9 siRNA transfected SiSo

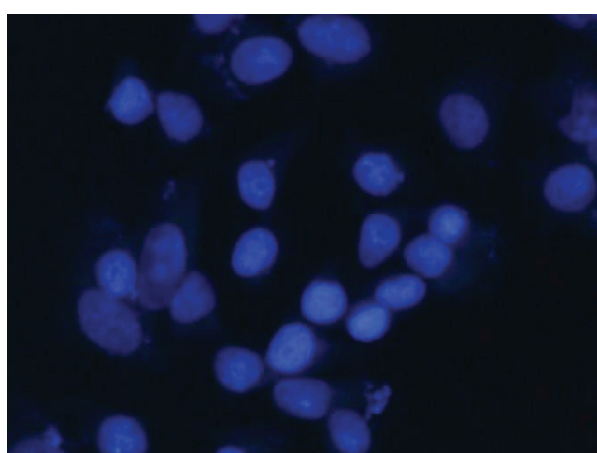

(B) MCF-7

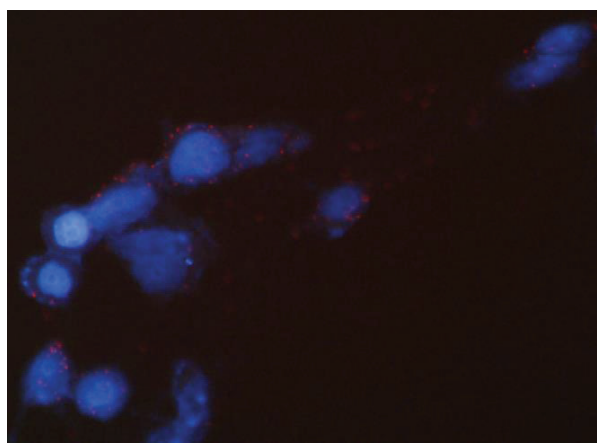

(D) RCAS1 siRNA transfected SiSo

(Original magnification: $\times 400$ )

(a)
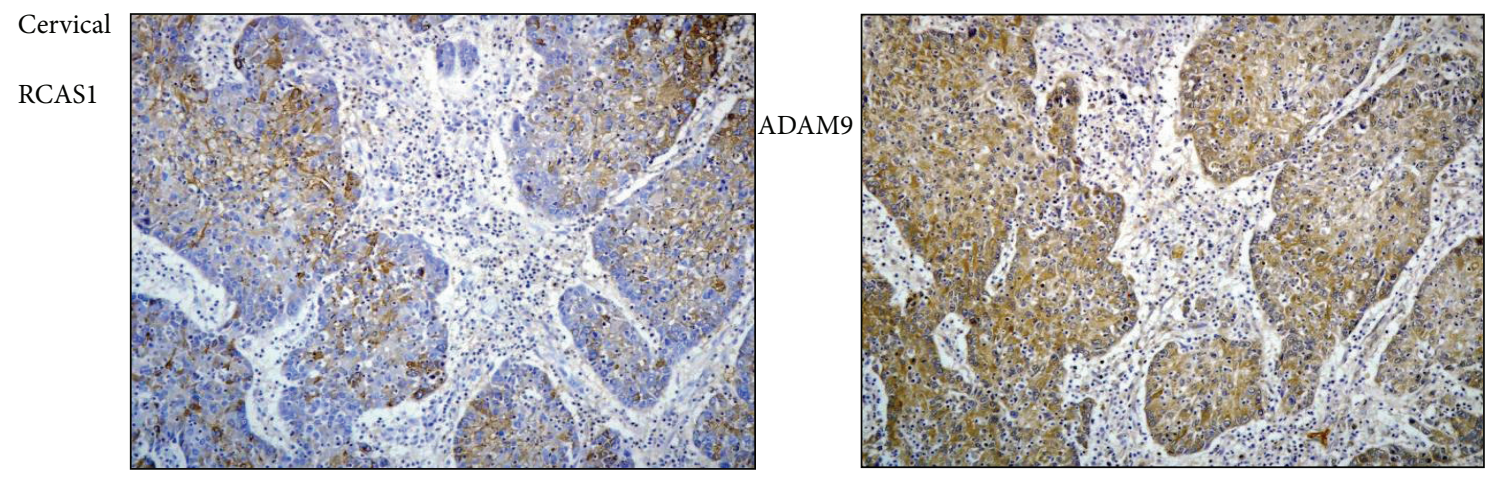

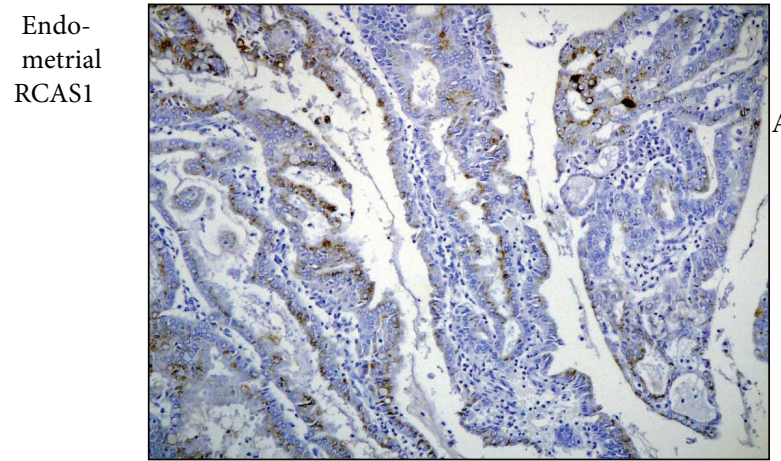

(b)

)

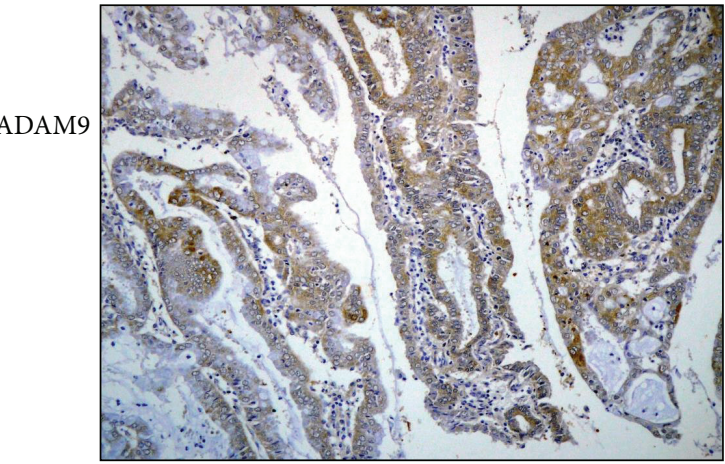

(Original magnification: $\times 100$ )

FIGURE 3: RCAS1 and ADAM9 expression in cell lines and cancerous tissues. (a) RCAS1 and ADAM9 colocalization was immunocytochemically analyzed in SiSo, MCF-7, ADAM9 siRNA-transfected SiSo, and RCAS1 siRNA-transfected SiSo cells using the Duolink detection kit. The red dots indicate RCAS1 and ADAM9 colocalization. (b) The expression pattern of RCAS1 and ADAM9 is shown in one representative case from cervical and endometrial cancer. Diffuse staining for RCAS1 and ADAM9 was observed both in the cytoplasm and on the cell membrane of the cancer cells. 
Cervical cancer $(n=47)$

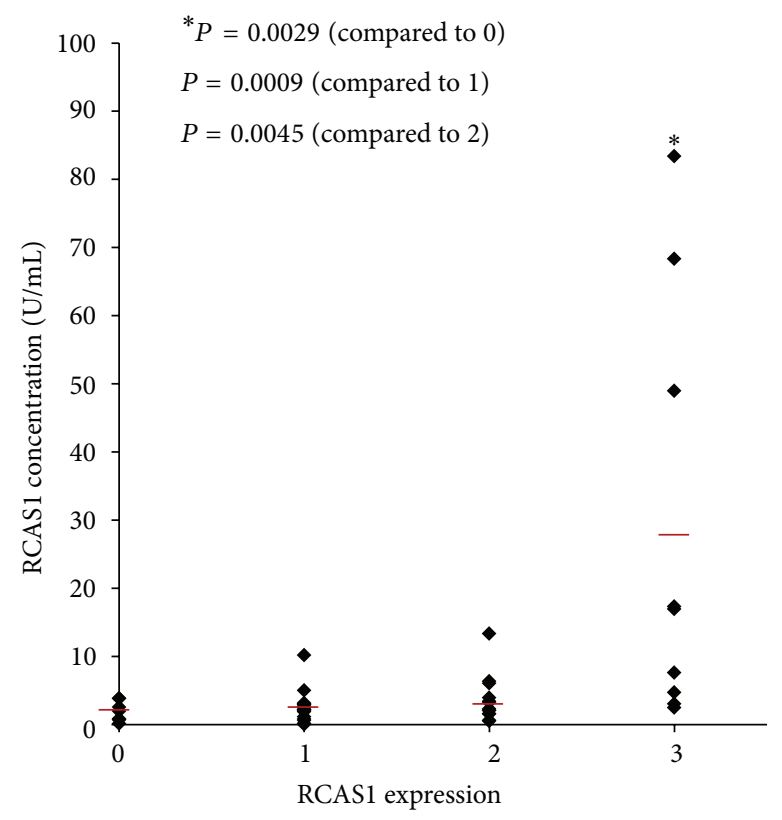

(a)
Endometrial cancer $(n=48)$

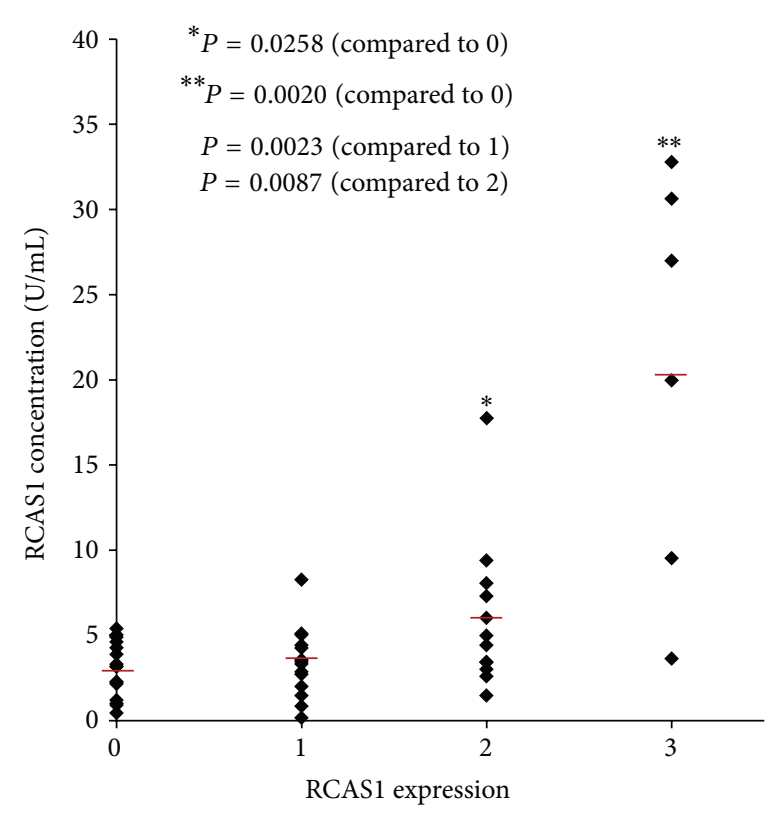

a)
Cervical cancer $(n=47)$

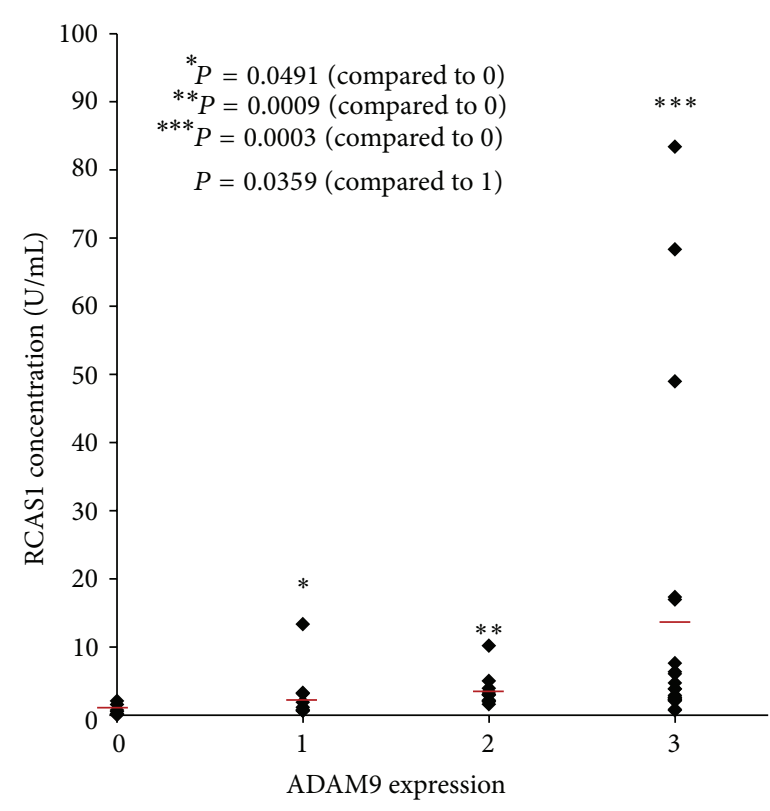

Endometrial cancer $(n=48)$

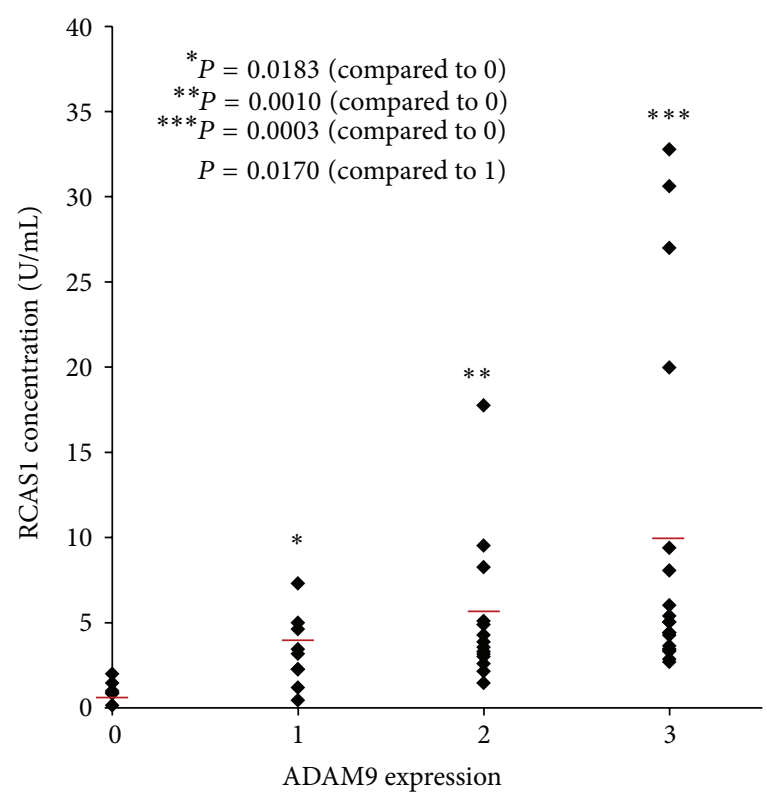

(b)

FIgURE 4: Association between serum RCAS1 concentration and RCAS1/ADAM9 expression in uterine cancer patients. Serum RCAS1 levels significantly increased in a manner that was dependent on both RCAS1 (a) and ADAM9 (b) expression in cervical and endometrial cancer patients. Mean values of triplicate measurements are shown.

Enhanced ADAM9 expression induced by gene transfection also promoted cell invasion [37]. ADAM9 is a secreted protein and its soluble form promoted the invasive phenotype of carcinoma cell lines by binding to the $\alpha 6 \beta 4$ and $\alpha 2 \beta 1$ integrins on the surface of carcinoma cells through its disintegrin domain [42]. In melanoma and a hepatic metastatic site of colon cancer, ADAM9 expression was upregulated at the invasion front, again supporting its role in tumor progression $[41,42]$.

Zubel et al. previously reported that ADAM9 is expressed in cervical squamous cell carcinoma [40]. In this study, the positive ratio of ADAM9 was $87 \%$ (29 out of 33 cases) and 
$78 \%$ (11 out of 14 cases) in squamous cell- and adenocarcinoma, respectively, which are values that are somewhat lower than the $93 \%$ (13 out of 14 cases) given in the previous report. On the other hand, this is the first report concerning ADAM9 expression in endometrial cancer wherein ADAM9 positivity was seen in $87 \%$ of cases (42 out of 48 cases) and ADAM9 expression was strong (3+) in 19 of 48 cases.

Although cancer patients currently receive multidisciplinary therapies that integrate surgery, radiation, and chemotherapy, limitations in the efficacy of anticancer treatments against advanced or recurrent tumors require the development of novel and highly specific targets for therapy. Considering the significance of tumor progression, RCAS1 has potential value as a unique biomarker and molecular target for diagnostics and therapy. Several therapeutic strategies should thus be considered to suppress the expression and function of RCAS1. A first strategy could be to modulate RCAS1 expression using siRNA. The value of this technique was shown in studies where the induction of molecularspecific siRNA into tumor cells reduced $T$ lymphocyte apoptosis and VEGF secretion, which was followed by tumor regression $[8,43]$. A second strategy for inhibiting RCAS1 function is to use antibodies. Serum from uterine and ovarian cancer patients inhibited growth of RCAS1 putative receptor expressing K562 cells, and this suppressive effect could be partially negated after immunoprecipitation to remove RCAS1 $[19,44]$. A third strategy would be to modulate ADAM9-mediated RCAS1 ectodomain shedding. Soluble RCAS1, rather than the membrane-anchored form, is mainly responsible for inducing apoptosis [9]. In this study, we did not use a catalytic inactive mutant form of ADAM9 to demonstrate that the proteolytic activity of ADAM9 is directly responsible for RCAS1 shedding. Thus it is possible that ADAM9 indirectly controls RCAS1 processing. To date, several methods have been reported to suppress ADAM9 expression and function. Knockdown of ADAM9 by RNA interference resulted in reduced cell proliferation, invasion, and metastasis $[35,39,45]$, as well as increased sensitivity to radiation and chemotherapeutic drugs [46]. Blocking of ADAM9 activity with specific antibodies resulted in inhibited cell growth of gastric cancer cell lines [34]. Reactive oxygen species (ROS) can also induce expression of ADAM9 via p38 mitogen-activated protein kinase activation [47]. Sung et al. observed apoptotic cell death in prostate cancer cells by decreasing ADAM9 expression via the administration of an antioxidant or genetic transfer of a hydrogen peroxide degradative enzyme [39]. Moreover, Moss et al. described that the metalloproteinase inhibitor marimastat is potent against ADAM9 [48].

Before initiating molecular targeting therapy, selection of eligible patients is necessary. For cervical and endometrial cancer in which RCAS1 is a clinical prognostic factor [2], tissue sampling and expression analysis of RCAS1 and ADAM9 can be easily performed. While several selective synthetic inhibitors that are active against a small number of ADAMs have recently been described $[49,50]$, adverse effects induced by targeting therapy can be a significant concern when the targeted molecules are ubiquitously expressed. Weskamp et al. generated mice lacking ADAM9 to learn more about the function of this protein during development and in adults [51]. During mouse development, ADAM9 mRNA is ubiquitously expressed, with particularly high expression levels in the developing mesenchyme, heart and brain. Despite the ubiquitous expression of ADAM9, these knockout mice appear to develop normally, are viable and fertile, and have no major pathological phenotypes compared to wild-type mice. Therefore, potential adverse effects produced by targeting ADAM9 activity could be anticipated to be tolerable.

Some recent advances might offer in the near future the opportunity to design such specific inhibitors using, for example, siRNAs or monoclonal antibodies. The precise understanding of the exact role played by RCAS1 and ADAM9 in cancer appears to be of particular importance from the perspective of designing new therapeutic strategies that are based on the control or inhibition of these proteins.

\section{Conclusion}

In several human malignancies, RCAS1 expression is associated with aggressive characteristics and poor overall survival. Since RCAS1 promotes tumor cell evasion of immune surveillance by inducing apoptosis in immune cells and also remodels the cancer stromal microenvironment, RCAS1 is believed to contribute to tumor progression. Soluble RCAS1, rather than the membrane-anchored form, is mainly responsible for inducing immune cell apoptosis. ADAM9 is involved in RCAS1 ectodomain shedding; therefore, inhibition of ADAM9 activity might contribute to controlling the biological functions of RCAS1. A precise understanding of the role played by RCAS1 and ADAM9 is essential to design novel strategies to treat cancer.

\section{Conflict of Interests}

The authors declare that there is no conflict of interests regarding the publication of this paper.

\section{Acknowledgments}

The authors thank Ms. Megumi Ito, Ms. Shoko Inagaki, Ms. Yuki Honda, Ms. Midori Sato, and Ms. Emiko Hori for technical assistance. This study was supported in part by a grant-in-aid for scientific research from the Japan Society for the Promotion of Science (no. 24592519) and Suzuken Memorial Foundation. This work was done in part at the Station for Collaborative Research and at the Morphology Core, Graduate School of Medical Sciences, Kyushu University.

\section{References}

[1] M. Nakashima, K. Sonoda, and T. Watanabe, "Inhibition of cell growth and induction of apoptotic cell death by the human tumor-associated antigen RCAS1," Nature Medicine, vol. 5, no. 8, pp. 938-942, 1999.

[2] K. Sonoda, "Novel therapeutic strategies to target RCAS1, which induces apoptosis via ectodomain shedding," Histology and Histopathology, vol. 26, no. 11, pp. 1475-1486, 2011. 
[3] K. Sonoda, M. Nakashima, T. Kaku, T. Kamura, H. Nakano, and T. Watanabe, "A novel tumor-associated antigen expressed in human uterine and ovarian carcinomas," Cancer, vol. 77, pp. 1501-1509, 1996.

[4] K. Sonoda, T. Kaku, T. Kamura, M. Nakashima, T. Watanabe, and H. Nakano, "Tumor-associated antigen 22-1-1 expression in the uterine cervical squamous neoplasias," Clinical Cancer Research, vol. 4, no. 6, pp. 1517-1520, 1998.

[5] K. Sonoda, T. Kaku, T. Hirakawa et al., "The clinical significance of tumor-associated antigen RCAS1 expression in the normal, hyperplastic, and malignant uterine endometrium," Gynecologic Oncology, vol. 79, no. 3, pp. 424-429, 2000.

[6] C. Giaginis, A. Giagini, and S. Theocharis, "Receptor-binding cancer antigen expressed on siso cells (RCAS1): a novel biomarker in the diagnosis and prognosis of human neoplasia," Histology and Histopathology, vol. 24, no. 6, pp. 761-776, 2009.

[7] M. Enjoji, K. Yamaguchi, M. Nakamuta et al., "Movement of a novel serum tumour marker, RCAS1, in patients with biliary diseases," Digestive and Liver Disease, vol. 36, no. 9, pp. 622-627, 2004.

[8] Y. Yoshida, K. Koga, T. Watanabe et al., "Potential utility of the tumour marker RCAS1 for monitoring patients with invasive extramammary Paget's disease," Acta Dermato-Venereologica, vol. 88, no. 3, pp. 296-297, 2008.

[9] K. Sonoda, S. Miyamoto, M. Nakashima, and N. Wake, "Receptor-binding cancer antigen expressed on SiSo cells induces apoptosis via ectodomain shedding," Experimental Cell Research, vol. 316, no. 11, pp. 1795-1803, 2010.

[10] Y. Izumi, M. Hirata, H. Hasuwa et al., "A metalloproteasedisintegrin, MDC9/meltrin- $\gamma / \mathrm{ADAM} 9$ and $\mathrm{PKC} \delta$ are involved in TPA-induced ectodomain shedding of membrane-anchored heparin-binding EGF-like growth factor," The EMBO Journal, vol. 17, no. 24, pp. 7260-7272, 1998.

[11] M. Golembo, E. Raz, and B. Shilo, “The Drosophila embryonic midline is the site of Spitz processing, and induces activation of the EGF receptor in the ventral ectoderm," Development, vol. 122, no. 11, pp. 3363-3370, 1996.

[12] J. G. Flanagan, D. C. Chan, and P. Leder, "Transmembrane form of the kit ligand growth factor is determined by alternative splicing and is missing in the SId mutant," Cell, vol. 64, no. 5, pp. 1025-1035, 1991.

[13] S. Davis, N. W. Gale, T. H. Aldrich et al., "Ligands for EPH-related receptor tyrosine kinases that require membrane attachment or clustering for activity," Science, vol. 266, no. 5186, pp. 816-819, 1994.

[14] H. Hua, M. Li, T. Luo, Y. Yin, and Y. Jiang, "Matrix metalloproteinases in tumorigenesis: an evolving paradigm," Cellular and Molecular Life Sciences, vol. 68, no. 23, pp. 3853-3868, 2011.

[15] N. Rocks, G. Paulissen, M. El Hour et al., "Emerging roles of ADAM and ADAMTS metalloproteinases in cancer," Biochimie, vol. 90, no. 2, pp. 369-379, 2008.

[16] R. Roy, J. Yang, and M. A. Moses, "Matrix metalloproteinases as novel biomarkers and potential therapeutic targets in human cancer," Journal of Clinical Oncology, vol. 27, no. 31, pp. 52875297, 2009.

[17] D. R. Edwards, M. M. Handsley, and C. J. Pennington, "The ADAM metalloproteinases," Molecular Aspects of Medicine, vol. 29, no. 5, pp. 258-289, 2009.

[18] K. Sonoda, M. Nakashima, T. Saito et al., "Establishment of a new human uterine cervical adenocarcinoma cell line, SiSo, and its: reactivity to anti-cancer reagents," International Journal of Oncology, vol. 6, no. 5, pp. 1099-1104, 1995.
[19] K. Sonoda, S. Miyamoto, T. Hirakawa et al., "Clinical significance of RCAS1 as a biomarker of uterine cancer," Gynecologic Oncology, vol. 103, no. 3, pp. 924-931, 2006.

[20] K. Sonoda, S. Miyamoto, A. Yamazaki et al., "Biologic significance of receptor-binding cancer antigen expressed on SiSo cells (RCAS1) as a pivotal regulator of tumor growth through angiogenesis in human uterine cancer," Cancer, vol. 110, no. 9, pp. 1979-1990, 2007.

[21] S. Mellberg, A. Dimberg, F. Bahram et al., "Transcriptional profiling reveals a critical role for tyrosine phosphatase VEPTP in regulation of VEGFR2 activity and endothelial cell morphogenesis," The FASEB Journal, vol. 23, no. 5, pp. 14901502, 2009.

[22] U. Lendeckel, J. Kohl, M. Arndt, S. Carl-McGrath, H. Donat, and C. Röcken, "Increased expression of ADAM family members in human breast cancer and breast cancer cell lines," Journal of Cancer Research and Clinical Oncology, vol. 131, no. 1, pp. 4148, 2005.

[23] M. J. Duffy, M. Mullooly, N. O’Donovan et al., “The ADAMs family of proteases: new biomarkers and therapeutic targets for cancer?" Clinical Proteomics, vol. 8, no. 1, article 9, 2011.

[24] M. Asai, C. Hattori, B. Szabó et al., "Putative function of ADAM9, ADAM10, and ADAM17 as APP $\alpha$-secretase," Biochemical and Biophysical Research Communications, vol. 301, no. 1, pp. 231-235, 2003.

[25] A. J. Huovila, A. J. Turner, M. Pelto-Huikko, I. Kärkkäinen, and R. M. Ortiz, "Shedding light on ADAM metalloproteinases," Trends in Biochemical Sciences, vol. 30, no. 7, pp. 413-422, 2005.

[26] M. J. Duffy, D. J. Lynn, A. T. Lloyd, and C. M. O'Shea, "The ADAMs family of proteins: From basic studies to potential clinical applications," Thrombosis and Haemostasis, vol. 89, no. 4, pp. 622-631, 2003.

[27] G. Weskamp, J. Krätzschmar, M. S. Reid, and C. P. Blobel, "MDC9, a widely expressed cellular disintegrin containing cytoplasmic SH3 ligand domains," The Journal of Cell Biology, vol. 132, no. 4, pp. 717-726, 1996.

[28] G. E. Olson, V. P. Winfrey, P. E. Matrisian, S. K. NagDas, and L. H. Hoffman, "Blastocyst-dependent upregulation of metalloproteinase/disintegrin MDC9 expression in rabbit endometrium," Cell and Tissue Research, vol. 293, no. 3, pp. 489-498, 1998.

[29] D. F. Seals and S. A. Courtneidge, "The ADAMs family of metalloproteases: multidomain proteins with multiple functions," Genes and Development, vol. 17, no. 1, pp. 7-30, 2003.

[30] V. Guaiquil, S. Swendeman, T. Yoshida, S. Chavala, P. A. Campochiaro, and C. P. Blobel, "ADAM9 is involved in pathological retinal neovascularization," Molecular and Cellular Biology, vol. 29, no. 10, pp. 2694-2703, 2009.

[31] Q. Xu, X. Liu, Y. Cai, Y. Yu, and W. Chen, "RNAi-mediated ADAM9 gene silencing inhibits metastasis of adenoid cystic carcinoma cells," Tumor Biology, vol. 31, no. 3, pp. 217-224, 2010.

[32] Y. Shintani, S. Higashiyama, M. Ohta et al., "Overexpression of ADAM9 in non-small cell lung cancer correlates with brain metastasis," Cancer Research, vol. 64, no. 12, pp. 4190-4196, 2004.

[33] C. O’Shea, N. McKie, Y. Buggy et al., "Expression of ADAM9 mRNA and protein in human breast cancer," International Journal of Cancer, vol. 105, no. 6, pp. 754-761, 2003.

[34] S. Carl-McGrath, U. Lendeckel, M. Ebert, A. Roessner, and C. Röcken, "The disintegrin-metalloproteinases ADAM9, ADAM12, and ADAM15 are upregulated in gastric cancer.," International journal of oncology, vol. 26, no. 1, pp. 17-24, 2005. 
[35] K. Tao, N. Qian, Y. Tang et al., "Increased expression of a disintegrin and metalloprotease-9 in hepatocellular carcinoma: implications for tumor progression and prognosis," Japanese Journal of Clinical Oncology, vol. 40, no. 7, Article ID hyq030, pp. 645-651, 2010.

[36] R. Grützmann, J. Lüttges, B. Sipos et al., "ADAM9 expression in pancreatic cancer is associated with tumour type and is a prognostic factor in ductal adenocarcinoma," The British Journal of Cancer, vol. 90, no. 5, pp. 1053-1058, 2004.

[37] J. Li, Z. Ji, C. Qiao, Y. Qi, and W. Shi, "Overexpression of ADAM9 promotes colon cancer cells invasion," Journal of Investigative Surgery, 2013.

[38] F. R. Fritzsche, K. Wassermann, M. Jung et al., "ADAM9 is highly expressed in renal cell cancer and is associated with tumour progression,” BMC Cancer, vol. 8, article no. 179, 2008.

[39] S. Sung, H. Kubo, K. Shigemura et al., "Oxidative stress induces ADAM9 protein expression in human prostate cancer cells," Cancer Research, vol. 66, no. 19, pp. 9519-9526, 2006.

[40] A. Zubel, C. Flechtenmacher, L. Edler, and A. Alonso, "Expression of ADAM9 in CIN3 lesions and squamous cell carcinomas of the cervix," Gynecologic Oncology, vol. 114, no. 2, pp. 332-336, 2009.

[41] P. Zigrino, C. Mauch, J. W. Fox, and R. Nischt, "ADAM9 expression and regulation in human skin melanoma and melanoma cell lines," International Journal of Cancer, vol. 116, no. 6, pp. 853-859, 2005.

[42] A. Mazzocca, R. Coppari, R. De Franco et al., "A secreted form of ADAM9 promotes carcinoma invasion through tumor-stromal interactions," Cancer Research, vol. 65, no. 11, pp. 4728-4738, 2005.

[43] Y. Han, W. Qin, and G. Huang, "Knockdown of RCAS1 expression by RNA interference recovers $\mathrm{T}$ cell growth and proliferation," Cancer Letters, vol. 257, no. 2, pp. 182-190, 2007.

[44] K. Sonoda, S. Miyamoto, F. Yotsumoto et al., "Clinical significance of RCAS1 as a biomarker of ovarian cancer," Oncology Reports, vol. 17, no. 3, pp. 623-628, 2007.

[45] K. C. Micocci, A. C. B. M. Martin, C. D. F. Montenegro et al., "ADAM9 silencing inhibits breast tumor cell invasion in vitro," Biochimie, vol. 95, no. 7, pp. 1371-1378, 2013.

[46] S. Josson, C. S. Anderson, S. Y. Sung et al., "Inhibition of ADAM9 expression induces epithelial phenotypic alterations and sensitizes human prostate cancer cells to radiation and chemotherapy," Prostate, vol. 71, no. 3, pp. 232-240, 2011.

[47] O. M. Fischer, S. Hart, A. Gschwind, N. Prenzel, and A. Ullrich, "Oxidative and osmotic stress signaling in tumor cells is mediated by ADAM proteases and heparin-binding epidermal growth factor," Molecular and Cellular Biology, vol. 24, no. 12, pp. 5172-5183, 2004.

[48] M. L. Moss, J. M. White, M. H. Lambert, and R. C. Andrews, "TACE and other ADAM proteases as targets for drug discovery," Drug Discovery Today, vol. 6, no. 8, pp. 417-426, 2001.

[49] M. L. Moss, A. Stoeck, W. Yan, and P. J. Dempsey, "ADAM10 as a target for anti-cancer therapy," Current Pharmaceutical Biotechnology, vol. 9, no. 1, pp. 2-8, 2008.

[50] N. B. Merchant, I. Voskresensky, C. M. Rogers et al., “TACE/ ADAM-17: a component of the epidermal growth factor receptor axis and a promising therapeutic target in colorectal cancer," Clinical Cancer Research, vol. 14, no. 4, pp. 1182-1191, 2008.

[51] G. Weskamp, H. Cai, T. A. Brodie et al., "Mice lacking the metalloprotease-disintegrin MDC9 (ADAM9) have no evident major abnormalities during development or adult life," Molecular and Cellular Biology, vol. 22, no. 5, pp. 1537-1544, 2002. 


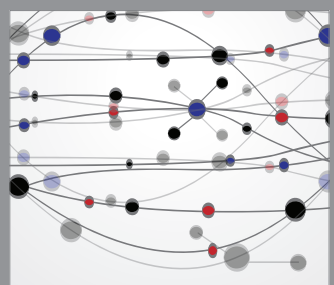

The Scientific World Journal
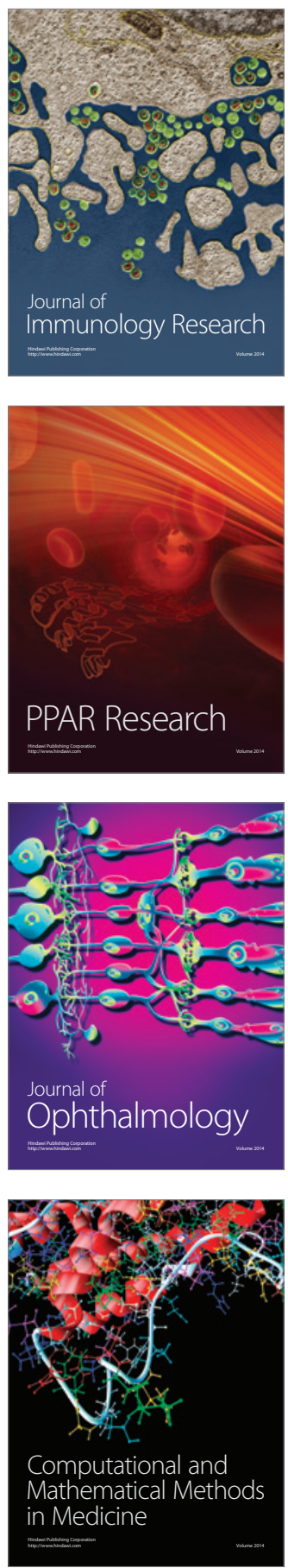

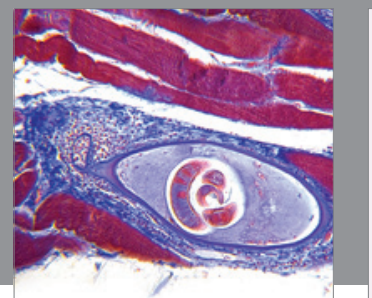

Gastroenterology

Research and Practice
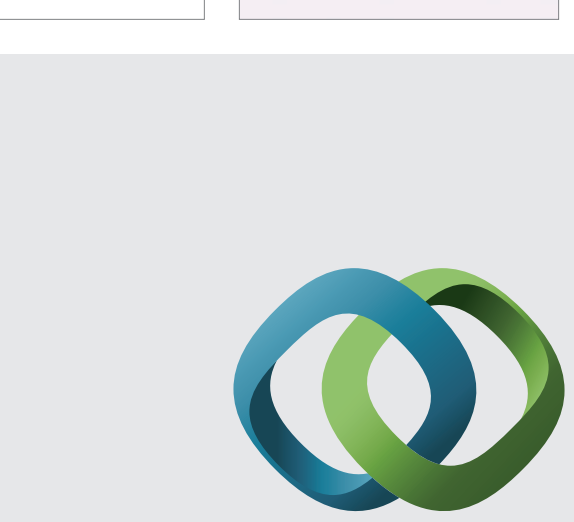

\section{Hindawi}

Submit your manuscripts at

http://www.hindawi.com
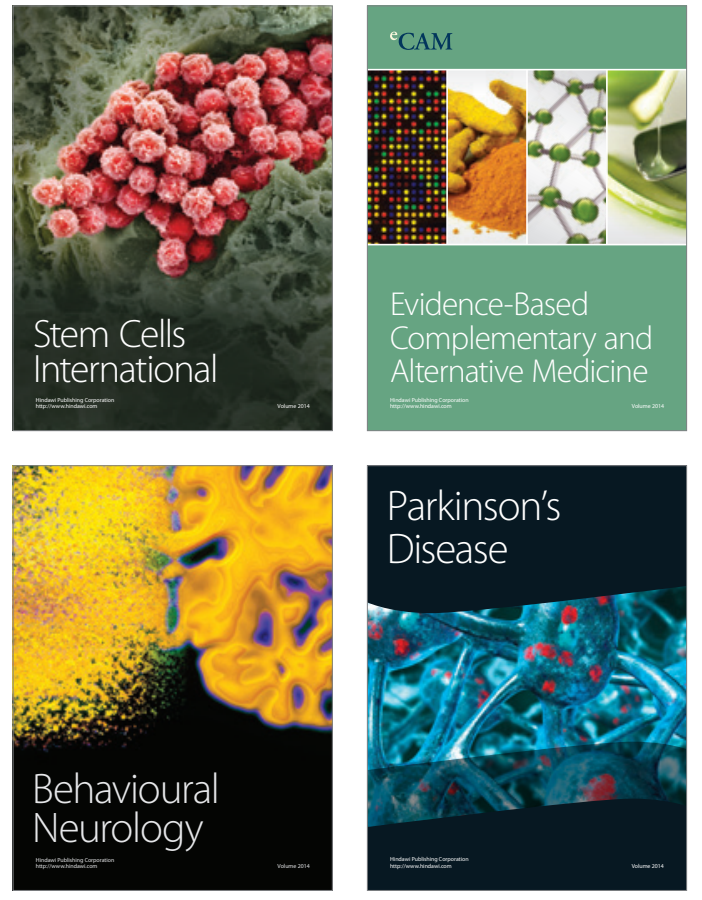
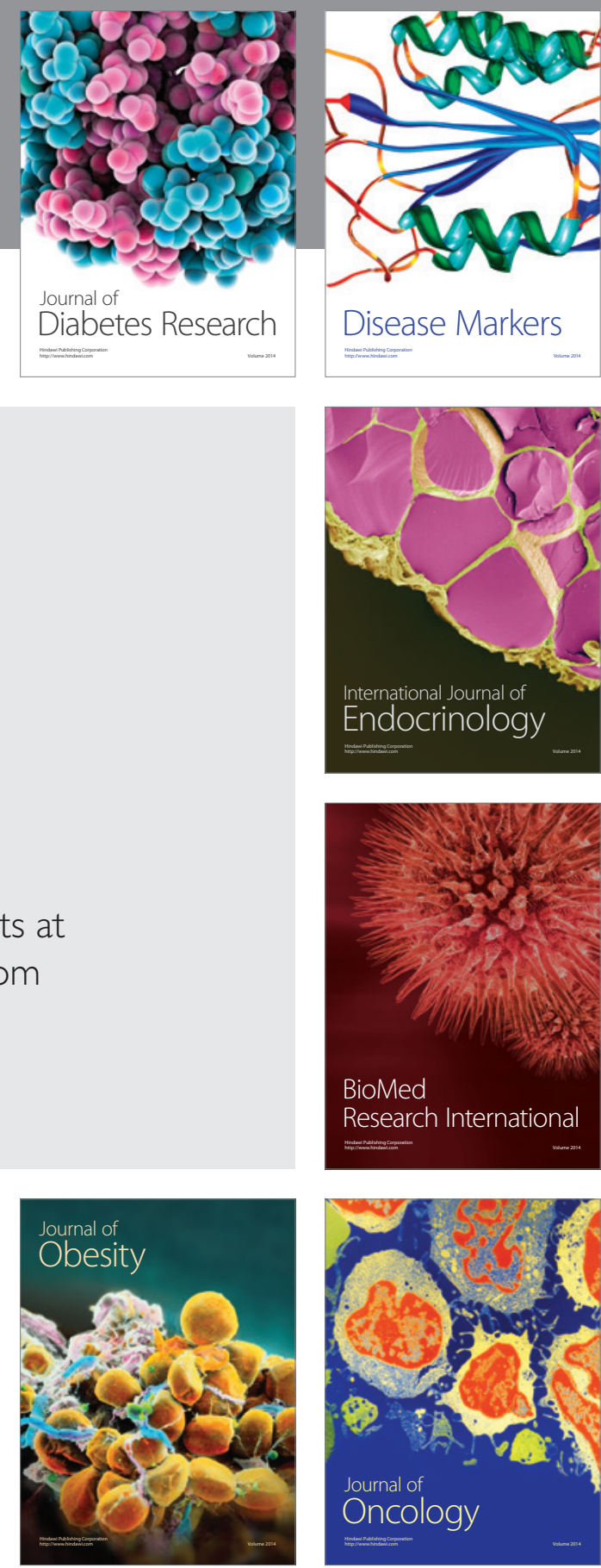

Disease Markers
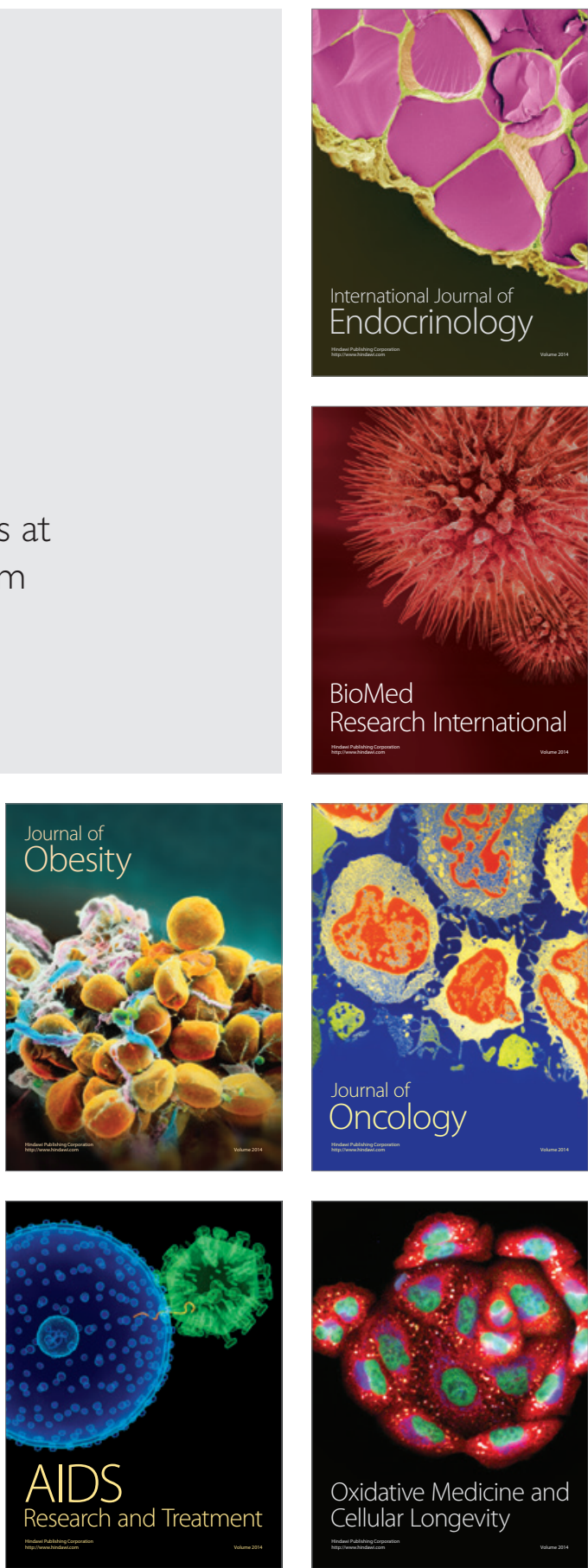